\title{
Predicción de Porcentaje de Masa Adiposa a través de Impedancia Bio-Eléctrica y Método Antropométrico
}

\author{
Mass Adipose Prediction Percentage through Biolectrical Impedance and Anthropometric Method
}

\author{
"Yuing, F. T. A; "Almagià, A. F.; "Lizana, P. J.; "Rodríguez, R. F. J.; "* Gallardo, L. R.; \\ ${ }^{* *}$ Nieto, C. F.; ${ }^{* *}$ Verdejo, S. A.; ${ }^{* * *}$ Ivanovic, D. M. \& ${ }^{* * * *}$ Binvignat,O.
}

YUING, F. T. A.; ALMAGIÀ, A. F.; LIZANA, P. J.; RODRIGUEZ, R. F. J.; GALLARDO, L. R.; NIETO, C. F.; VERDEJO, S. A.; IVANOVIC, D. M. \& BINVIGNAT, O. Predicción de porcentaje de masa adiposa a través de impedancia bio-eléctrica y método antropométrico. Int. J. Morphol., 30(3):872-876, 2012.

RESUMEN: Este estudio fue desarrollado con la finalidad de formular una ecuación de predicción de \% de masa adiposa en valores antropométricos, a partir, de los valores predeterminados a través del instrumento de bioimpedancia TANITA. Esto basado en la premisa de que cada día ha aumentado la importancia sobre la estimación de la masa adiposa a nivel clínico, por lo cual se evaluó una población de 28 sujetos cuyo rango de edad fluctúa entre los 20-28 años, pertenecientes a la Escuela de Caballería Blindada de la ciudad de Quillota. Se realizaron las mediciones pertinentes para la estimación de masa adiposa a través de la bioimpedancia y la antropometría, cuyos resultados arrojaron diferencias significativas entre ambos métodos, obteniendo un coeficiente de correlación de Pearson de 0,34. Esto nos indica que al evaluar los criterios de homocedasticidad, linealidad, para generar una ecuación de estimación, no sería posible.

PALABRAS CLAVE: Antropometría; Bioimpedancia eléctrica; Ecuación de Predicción.

\section{INTRODUCCIÓN}

En la actualidad, existen diversidad de modelos, métodos y técnicas para la evaluación de la composición corporal. Esta última representa una parte esencial en la valoración del estado nutritivo de un individuo y consiste en el fraccionamiento de la masa total del cuerpo en sus distintos componentes principales. Según el número de elementos en que se divide el peso total se han desarrollado ecuaciones que van desde el modelo más sencillo, bi compartimental o de dos componentes, que solo considera masa grasa y masa magra, hasta los más complejos que distinguen hasta cinco o seis compartimentos, incluyendo dentro de estos a la masa ósea, masa muscular, masa grasa, piel y masa residual.

Los métodos del análisis de impedancia bioeléctrica y antropometría poseen características similares en cuanto a accesibilidad y fácil manejo, son reproducibles y presentan facilidad para ser aceptados por el paciente como una técnica normal de examen, además son métodos de campo doblemente indirectos que emplean ecuaciones individua- les de predicción, de acuerdo con la edad, el sexo y el nivel de actividad física del individuo evaluado.

Una vez conocida la necesidad de determinar la grasa corporal por ambos métodos de medición, es que a continuación se plantea el objetivo de estudio, el objetivo general, objetivos específicos, planteamiento del problema, entre otros. Sumado a lo anterior realizaremos una revisión bibliográfica, la cual nos apoyara en la ejecución de nuestro estudio y por medio del análisis de los datos y las conclusiones conoceremos si dicho estudio, no realizado en Chile, obtiene sus frutos.

\section{SUJETOS Y MÉTODO}

Los sujetos evaluados deberán pertenecer a la Escuela de Caballería Blindada, ser funcionarios de planta, de sexo

\footnotetext{
Laboratorio de Antropología Física y Anatomía Humana, Instituto de Biología, Facultad de Ciencias, Pontificia Universidad Católica de Valparaíso, Valparaíso, Chile.

*** Escuela de Kinesiología, Universidad del Mar, Chile.

*** Instituto de Nutrición y Tecnología de los Alimentos, Universidad de Chile, Santiago, Chile.

***** Profesor colaborador Laboratorio de Antropología Física y Anatomía Humana, Instituto de Biología, Facultad de Ciencias, Pontificia Universidad Católica de Valparaíso. Chile. Centro Anatómico, Campus Talca, Universidad Autónoma, Talca, Chile.
} 
masculino, mayores de 20 años y menores de 28 , que practiquen actividad física como mínimo hace 2 meses (3 veces por semana) y previo a la medición, que no presenten ninguna condición patológica que impida la realización de la misma.

Se evaluaron 28 sujetos de planta de una población total de 200 individuos, cuyo rango de edad fluctúa entre los 20 y 28 años. Estos individuos corresponden al 14\% de la población total de planta, la cual lleva más de dos meses realizando actividad física por lo menos 3 veces por semana.

Se realizó la evaluación por estaciones. En la primera de ellas se registraban sus datos personales, en la segunda se evaluaba la estatura, en la tercera el registro de peso corporal y porcentaje de masa adiposa por medio de Impedancia Bioeléctrica y por último en la cuarta estación se realizó la medición antropométrica de los sujetos, en donde todas las mediciones fueron realizadas en el hemicuerpo derecho. En primer lugar se realizó el reconocimiento y marcación de los puntos de referencia anatómicos (con lápiz demográficos negro), seguido de la medición de pliegues cutáneos, posterior a esto se realizó la medición de perímetros y por último la medición de diámetros y registro de todo lo evaluado.

Este estudio de tipo paramétrico no experimental, presenta como principal variable estudiada el porcentaje de masa adiposa, el cual es determinada por dos métodos distintos de medición, por un lado, el instrumento TANITA de bioimpedancia eléctrica y por el otro, a través del método antropométrico, utilizando el modelo propuesto por Deborah Kerr (Kerr, 1988).

Después de realizar el registro de todos los datos obtenidos gracias a las mediciones realizadas, se toman los resultados arrojados por el método de bioimpedancia eléctrica y se realizan los cálculos para la separación de la composición corporal en dos compartimentos, es decir, en Masa Libre de Grasa (MLG) y Masa Grasa (MG). Por otra parte, a través de la utilización de la ecuación antropométrica de Deurenberg et al. (1991), se obtiene el valor específico de Impedancia para cada sujeto evaluado, arrojando la cantidad de resistencia que presenta el tejido, al paso de la corriente eléctrica. Dicha ecuación fue utilizada para el desarrollo de este estudio en la obtención de porcentaje de masa adiposa a través del método de BIA, a partir, de los valores ya obtenidos como la MLG (masa magra), la estatura y peso.

Con el objetivo de generar una ecuación, todos los porcentajes de masa adiposa obtenidos por ambos métodos de los 28 sujetos evaluados, fueron ingresados en un gráfico de dispersión, con el objetivo de verificar si es posible la obtención de puntos agrupados lo más cercano a la formación de una recta. De esta manera comprobaríamos de una vez, si los datos cumplen con la linealidad y homogeneidad.

\section{RESULTADOS}

En la Tabla I se presentan las características generales de los participantes, en donde se puede apreciar la edad promedio que es de $26,8( \pm 1,4)$ y cuyos porcentajes de masa adiposa fluctúan entre 22,3 a $23,7 \%$ para BIA y antropometría respectivamente. Por otro lado, se puede apreciar que la diferencia entre medias para los porcentajes de masa adiposa a través de BIA, como la del método antropométrico, es mínima, siendo una razón del 5,8\%. De este dato, podemos inferir a simple vista, que ambos métodos podrían ser correlaciónales, ya que como mencionamos anteriormente la diferencia es escasa, pero si nos detenemos a observar sus desviaciones estándar, apreciamos la gran diferencia de los datos, llegando a ser esta diferencia casi el doble por parte del método de BIA respecto al método antropométrico. Deducimos de esto último que, los resultados de la BIA son muy dispares entre sus homólogos.

Tabla I. Datos generales de los participantes del estudio.

\begin{tabular}{lc}
\hline Variables & Resultados \\
\hline Número de personas & 28 \\
Edad & $26,8 \pm 1,4$ \\
Peso & $75,5 \pm 10,3 \mathrm{~kg}$ \\
Talla & $169,4 \pm 5,0 \mathrm{~cm}$ \\
$\%$ BIA & $22,3 \pm 6,8$ \\
MLG en kg & $57,0 \pm 4,9$ \\
MG en kg & $16,2 \pm 7,5$ \\
Impedancia en ohm & $398,5 \pm 74,7$ \\
\% Antropometría & $23,7 \pm 3,5$ \\
\hline
\end{tabular}

En la Tabla II se observan los datos estadísticos para la realización del ajuste de regresión y los resultados de la misma. Cabe destacar que previo a la regresión, la desviación estándar que presentaba la variable X pasa de un 6,88 a un valor de 1,061, lo cual nos indica que hubo una aproximación importante entre los resultados, con los que podríamos cumplir los criterios necesarios para la realización de la ecuación de regresión que se pretende formular.

En la Figura 1 con $2( \pm \mathrm{DE})$, muestra los límites dentro de los que se encuentran la mayoría de los sujetos post ajuste, esta no arroja diferencias significativas con el gráfico pre-ajuste. Por lo cual, se reafirma que no hay cumpli- 
YUING, F. T. A.; ALMAGIÀ, A. F.; LIZANA, P. J.; RODRIGUEZ, R. F. J.; GALLARDO, L. R.; NIETO, C. F.; VERDEJO, S. A.; IVANOVIC, D. M. \& BINVIGNAT, O. Predicción de porcentaje de masa adiposa a través de impedancia bio-eléctrica y método antropométrico. Int. J. Morphol., 30(3):872-876, 2012.

Tabla II. Resultados de variables consideradas y sus respectivos rangos.

\begin{tabular}{lcccc}
\hline Parámetros & \% BIA & \% & \% BIA teórico & BIA (*) \\
\hline- & 687 & 682,73 & 902,72 & 682,31 \\
$X$ & 24,53 & 24,31 & 27,13 & 24,23 \\
DE & 6,88 & 3,54 & 12,96 & 1,06 \\
\hline
\end{tabular}

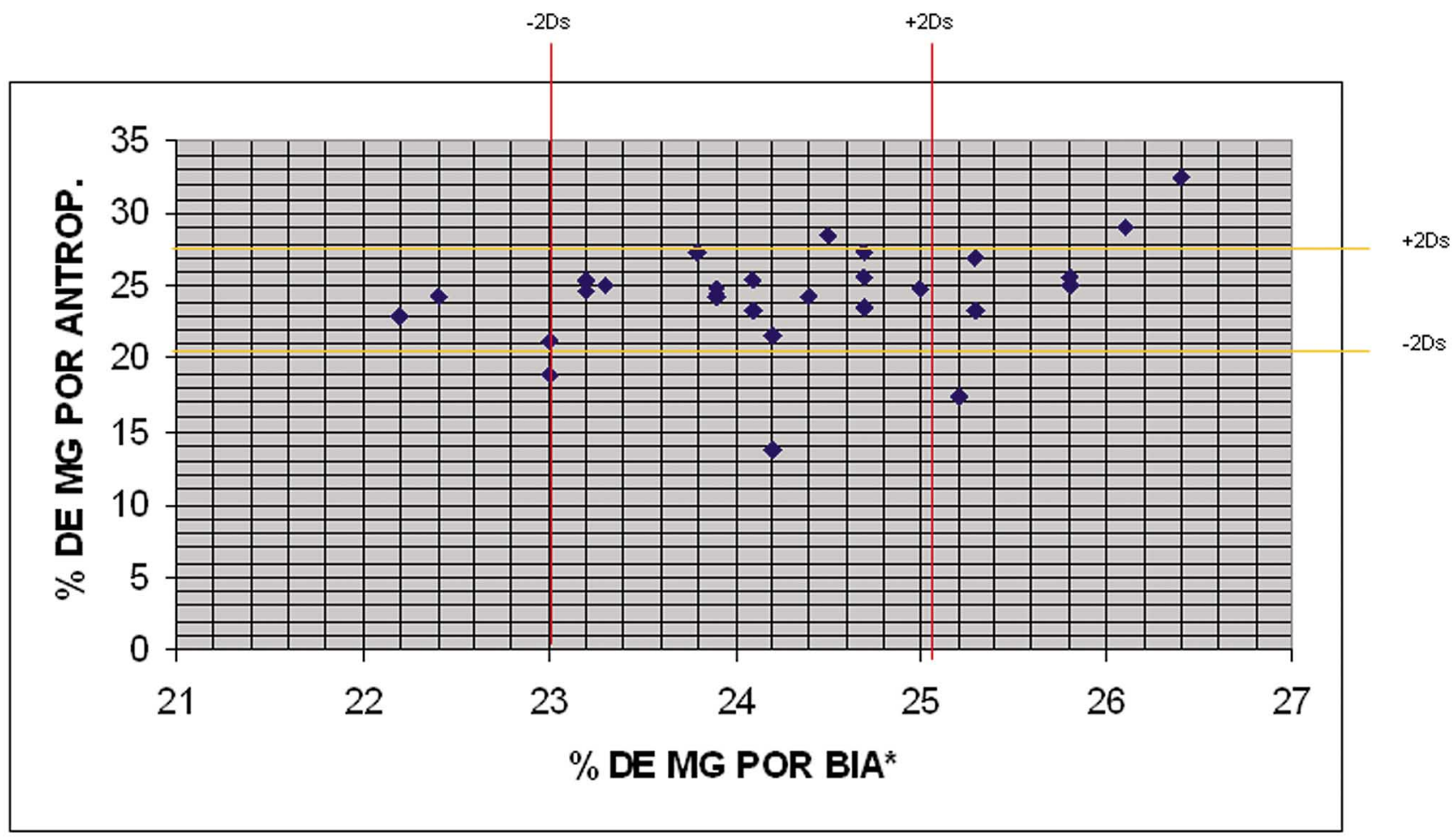

Figura 1. Gráfico de dispersión de Porcentaje de masa adiposa ( \pm 2 ds) post-ajuste.

miento de los criterios básicos para el desarrollo de una ecuación de regresión.

Debido a los resultados obtenidos, cabe destacar, que muchos autores apoyan el hecho de que el análisis de impedancia bioeléctrica por la técnica pie-pie, es de menor valor de predicción que la técnica mano-pie. Esto se debe a que en la primera técnica, la corriente eléctrica atraviesa principalmente el segmento inferior del cuerpo y el superior es subvalorado, además de lo anterior, la posición bípeda puede provocar acumulación de líquidos en las extremidades inferiores y así alterar por consecuencia los valores arrojados por la BIA.

Por otra parte, otro factor que pudo haber sido desfavorable en la creación de la ecuación, fue habernos basado en fórmulas o principios de Deborah Kerr. Estas fórmulas plantean una forma compleja de valorar la composición cor- poral, mientras que existe otra ecuación que es tan válida como la anterior, para calcular el porcentaje de masa grasa, nos referimos a la ecuación de Siri. Incluso la SEEDO (Sociedad Española para el Estudio de la Obesidad), recomienda la utilización de los pliegues cutáneos y la ecuación de Siri para la valoración del porcentaje de grasa corporal, dando por válidas tanto la forma global como la forma específica de calcular la densidad corporal con la ecuación de Durning/ Womersley. De esta manera se pretendía homologar las ecuaciones de BIA y de SIRI, obteniendo posteriormente la ecuación anhelada. El problema que se presentó, a parte de los resultados con diferencias significativas, fue que no se midió el pliegue bicipital, el cual está incluido en la mayoría de las ecuaciones antropométricas para la predicción del porcentaje de masa adiposa, por lo tanto, se excluyó todas aquellas ecuaciones que utilizaban el pliegue bicipital, limitando la creación de nuestra ecuación de predicción. 


\section{DISCUSIÓN}

Si bien, el objetivo general del estudio era establecer una correlación entre los porcentajes de masa adiposa, obtenidos por Impedancia Bioeléctrica y Antropometría, a través del desarrollo de una posible ecuación de predicción, que sea objetiva y confiable, nos llama profundamente la atención la gran disparidad de los resultados de masa grasa obtenido entre ambos métodos. Dichos resultados arrojan diferencias significativas entre ambos métodos, lo cual sugiere que éstos no son similares ni homogéneos. Esto se pudo observar en el gráfico de correlación, en donde la nube de puntos resultantes no se dispuso de manera paralela al eje de las abscisas, por ende, no hubo distribución aleatoria a ambos lados de la recta. Producto de esta escasa linealidad, es que la variable de porcentaje de masa grasa por BIA de cada sujeto, se somete a transformación para evitar errores de estimación en la supuesta futura ecuación, mediante el coeficiente de correlación lineal de Pearson, el que arroja un resultado de 0,34. Esto quiere decir, que hubo un ajuste en cuanto al resultado de las variables, pero no fue lo realmente significativo para la creación de una ecuación fiable.

Las grandes diferencias o la poca correlación entre los métodos descritos, pueden provenir del supuesto distinto en que se basan. El método antropométrico estima la grasa corporal y la masa libre de grasa, a partir, de la sumatoria de pliegues cutáneos, mientras que la BIA estima el contenido de agua corporal total, a partir, del índice de bioimpedancia para así poder calcular los porcentajes de grasa y los libres de grasa.

También debemos considerar que la composición corporal varía según las características raciales de los sujetos, por lo tanto, es necesario e importante considerar, que las ecuaciones de BIA podrían no ser aplicables a la población chilena, sobre todo en este estudio realizado, ya que sólo involucró sujetos de sexo masculino y de un determinado rango de edad, lo cual no es representativo de la po- blación en general. Además con respecto a las ecuaciones de BIA, se consultó a Balanzas eléctricas TANITA para solicitar información sobre la ecuación utilizada por dicho modelo y esa información fue negada por parte de la institución, por lo tanto, se decidió utilizar la Ecuación de Deuremberg et al., ya que ésta se basa en variables que coinciden con las tomadas en este estudio, arrojando valores distintos a los arrojados por la BIA TANITA, por lo tanto, podemos deducir que quizás la ecuación realizada no fue la más indicada. Esto lo corroboramos al observar la media obtenida del porcentaje de masa grasa según el instrumento TANITA y el porcentaje teórico dado por la ecuación anteriormente mencionada, en donde esta última sobreestima en un $10,5 \%$ el porcentaje de masa adiposa en la media de la población, por lo tanto, esto nos da a entender que posiblemente no es la ecuación utilizada por la BIA TANITA.

Debido a todo lo anterior, podemos concluir que la hipótesis que se confirma a través de los resultados estadísticos y a partir de los valores obtenidos por impedancia bioeléctrica y antropometría, no se puede generar una ecuación de estimación de porcentajes de masa grasa, ya que los resultados obtenidos por ambos métodos, presentan diferencias significativas, las cuales originarían una ecuación que no cumple con los criterios de linealidad homocedasticidad, normalidad, valores típicos, por lo tanto, no sería una ecuación de predicción válida.

AGRADECIMIENTOS. A cada una de las personas participantes de la investigación, a la Escuela de Caballería Blindada de la ciudad de Quillota por la autorización y facilidades para la realización de la misma y a cada uno del grupo de trabajo del Laboratorio de Antropología Física y Anatomía Humana de la Pontificia Universidad Católica de Valparaíso, por la constancia y colaboración en el trabajo. Por otro lado agradecer a la Dirección de Investigación, Vicerectoría de Investigación y Estudios Avanzados e Instituto de Biología de la Facultad de Ciencias. Pontificia Universidad Católica de Valparaíso. Chile, por su constante apoyo en materia investigativa.

YUING, F. T. A.; ALMAGIÀ, A. F.; LIZANA, P. J.; RODRIGUEZ, R. F. J.; GALLARDO, L. R.; NIETO, C. F.; VERDEJO, S. A.; IVANOVIC, D. \& BINVIGNAT, O. Mass adipose prediction percentage through biolectrical impedance and anthropometric method. Int. J. Morphol., 30(3):872-876, 2012.

SUMMARY: This case was developed with the objective of makes a prediction equation of adipose mass percent in values anthropometric, to star off the predetermined slant of instruments of impedance bioelectric TANITA. This is based in the premise that every day the importance of estimation about the fat mass in a clinic level, it is getting bigger and bigger, for this reason a population of twenty eight people with age range fluctuate among twenty to twenty eight was evaluated, they were part of "Escuela de Caballería Blindada" in the city of Quillota. The Pertinent measurements for the estimation of fatty mass were realized through of impedance bioelectric and anthropometry, the results made big differences between both methods, obtaining an index of correlation coefficient a 0.13 and a person correlation of 0.34 . This suggests that possible creation does not accomplish with the criteria homescedasticity, in brief, this is unsafe.

KEY WORDS: Anthropometry; Impedance bioelectric; Equation of prediction. 
YUING, F. T. A.; ALMAGIÀ, A. F.; LIZANA, P. J.; RODRIGUEZ, R. F. J.; GALLARDO, L. R.; NIETO, C. F.; VERDEJO, S. A.; IVANOVIC, D. M. \& BINVIGNAT, O. Predicción de porcentaje de masa adiposa a través de impedancia bio-eléctrica y método antropométrico. Int. J. Morphol., 30(3):872-876, 2012.

\section{REFERENCIAS BIBLIOGRÁFICAS}

Deurenberg, P.; Weststrate, J. A. \& Seidell, J. C. Body mass index as a measure of body fatness: age- and sex-specific prediction formulas. Br. J. Nutr., 65(2):105-14, 1991.

Kerr, D. A. An anthropometric method for the fractionation of skin, adipose, muscle, bone and residual tissue masses in males and females age 6 to 77 years. M. Sc. Thesis. British Columbia, Simon Fraser University, 1988.

\author{
Dirección para correspondencia: \\ Atilio Aldo Almagià Flores \\ Laboratorio de Antropología Física y Anatomía Humana \\ Laboratorio de Técnicas Anatómicas \\ Instituto de Biología \\ Avenida Universidad 330 \\ Curauma, Placilla \\ Pontificia Universidad Católica de Valparaíso \\ Valparaíso \\ CHILE
}

Email: aalmagia@gmail.com aalmagia@ucv.cl

Recibido : 14-05-2012

Aceptado: 22-06-2012 